\title{
Analysis of Influence of Modes \\ for Wire Saws Efficient Extraction \\ of Natural Stone Blocks
}

\author{
Gennadiy D. Pershin and Maxim S. Ylyakov* \\ Magnitogorsk State Technical University \\ named after G.I. Nosov \\ 38 Lenina, Magnitogorsk, 455000, Russia
}

Received 05.03.2015, received in revised form 19.09.2015, accepted 06.10.2015

The dependences of the main parameters of the cutting process (performance, power consumption and diamond tools) on the mode of wire saw at different heights quarry face. The technique of choosing the rational mode control cable saw, taking into account the dependence of performance established branch of the monolith from the array and operating costs for his department, depending on the height of the ledge. To be able to select the sound mode control cable saw proposed a comprehensive technical and economic indicators, characterized costs charged to the intensity of space capacity of stone from the array.

Keywords: wire saw, performance, consumption tool, the specific work of cutting costs.

DOI: $10.17516 / 1999-494 X-2015-8-7-928-940$.

\section{Анализ влияния режимов работы \\ канатных пил на эффективность добычи \\ блоков природного камня}

\author{
Г.Д. Першин, М.С. Уляков \\ Магнитогорский государственный \\ технический университет им. Г.И. Носова \\ Россия, 455000, Магнитогорск, Ленина, 38
}

Приведены зависимости основных показателей процесса резания (производительность, расход энергии и алмазного инструмента) от режима работы канатной пиль при различной высоте добычного уступа. Разработана методика выбора раџионального режима управления канатной пилой, учитывающая установленные зависимости производительности отделения монолита от массива и эксплуатационные затраты на его отделение в зависимости от

(c) Siberian Federal University. All rights reserved

* Corresponding author E-mail address: maxim-atlet@yandex.ru 
высоты уступа. Для возможности выбора рационального режима управления канатной пилой предложен комплексный технико-экономический показатель, характеризуемый затратами, отнесенными к интенсивности отделения объемов камня от массива.

Ключевые слова: канатная пила, производительность, расход инструмента, удельная работа резания, себестоимость.

\section{Введение}

Добычные горные машины с гибким режущим инструментом к настоящему времени на каменных карьерах всего мира получили доминирующее положение. Это связано с возмож-

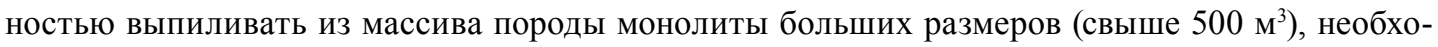
димость в которых обусловлена повышением выхода товарных блоков на трещиноватых месторождениях. Применение на данных машинах канатно-алмазного инструмента позволило вести добычные работы и на высокопрочных месторождениях природного камня (породы типа гранит). Разработка и применение на карьерах канатно-алмазных пил (такое название утвердилось у горняков) взамен ранее применяемых канатно-абразивных пил, где в качестве абразивной пульпы использовался кварцевый песок с водой, позволило повысить производительность резания в десять и более раз на породах средней прочности и расширить диапазон прочности добываемого камня до уровня гранитов, долеритов и т. д.

К началу XXI в. канатно-абразивные пилы безвозвратно ушли в прошлое, поэтому нет необходимости современные горные машины с гибким режущим рабочим органом называть как канатно-алмазные пилы, выделяя алмазный инструмент по отношению к абразивному, который уже не применяется в настоящий период времени.

Отделение больших по объему монолитов камня предполагает применение высокоуступной технологии отработки месторождения, когда все линейные параметры монолита обосновываются по условию максимального выхода товарных блоков в зависимости от природной трещиноватости массива. Независимо от прочности камня теоретическими исследованиями и практикой обоснована двухстадийная схема добычи товарных блоков, когда на первой, самой трудоемкой стадии отделяется монолит обоснованных размеров с помощью канатных пил (КП). На второй стадии монолит разделывается на товарные блоки различными способами и реализующими их техническими средствами в зависимости от прочности камня. Для пород типа мрамор применяют баровые камнерезные машины либо пассировочные КП для окварцованных месторождений. На породах типа гранит используется шпуровой способ с применением клиньев (механических, гидравлических), а также невзрывчатых разрушающих составов (HPC).

Цель данной работы заключается в исследовании влияния режимов работы КП на основные технико-экономические показатели процесса отделения монолитов камня от породного массива.

\section{Основная часть}

На технико-экономические показатели отделения монолита от массива влияют его линейные размеры $(H, L, B)$, режимы работы КП и прочность камня [1-4]. Экономическая оценка работы КП производится с учетом производительности резания, удельного расхода электро- 
энергии и алмазного инструмента. Удельные эксплуатационные затраты (руб/м²) на отделение монолита от массива с помощью КП при этом определяются по зависимости

$$
C_{S}=\frac{C_{0}}{K_{\text {ио }} \cdot \Pi}+b \cdot A \cdot C_{э}+b \cdot R \cdot \gamma_{\mathrm{a}} \cdot C_{\text {и }},
$$

где $C_{0}, C_{э}, C_{\text {и }}$ - стоимости соответственно работы канатной пилы (руб/ч), электроэнергии (руб/ кВт·ч), алмазного инструмента (руб/карат); $K_{\text {ио }}=0,75$ - расчетный коэффициент использования КП во времени; П - техническая производительность КП, $\mathrm{M}^{2} / \mathrm{c} ; b$ - ширина пропила (диаметр алмазорежущей втулки гибкого инструмента), м; $A$ - удельная работа резания, Дж/м³ $R$ - удельный расход алмазного инструмента, $\mathbf{M}^{3} / \mathbf{M}^{3} ; \gamma_{\mathrm{a}}$ - содержание алмазов в единице объема алмазонесущего слоя инструмента, карат/м³

Первое слагаемое уравнения представляет затраты на амортизационные отчисления камнерезного оборудования с учетом затрат на обслуживание и ремонт и заработную плату оператору КП $C_{\mathrm{AO}+3 п}$; второе слагаемое - это затраты на электроэнергию, потребляемую двигателем

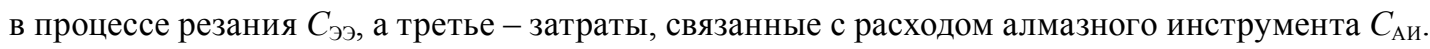

Применяются две схемы управления работой КП. В первой схеме через задание на пульте управления силы тока определенной величины выдерживается постоянной мощность главного привода $\left(N=\right.$ const), но изменяется скорость подачи тележки машины ( $\left.V_{n}=\mathrm{var}\right)$. Существующая обратная связь отслеживает изменение мощности энергии основного двигателя и сводит это изменение к нулю за счет увеличения или уменьшения скорости вращения двигателя механизма подачи. С изменением скорости подачи прямо пропорционально изменяется и силовой режим резания, т. е. величина контактного давления инструмента на породу ( $\left.\sigma_{n}=\mathrm{var}\right)$. Во второй схеме тележке КП путем использования реостата в электрической схеме привода подачи задается постоянная скорость перемещения $\left(V_{n}=\mathrm{const}\right)$. При этом на пульте управления амперметр показывает силу тока на главном приводе машины. Так как в режиме постоянной скорости подачи обеспечивается неизменным контактное давление инструмента на породу ( $\sigma_{n}=$ const), то величина силы тока будет изменяться прямо пропорционально изменению длины контакта инструмента с породой, что вызовет аналогичное изменение мощности энергии главного привода КП.

Как видим, применяемые схемы управления работой КП влияют только на силовой режим резания. По первой схеме силовой режим переменный во времени, во второй схеме - постоянный. Силовой режим определяет и производительность резания, и мощность главного привода камнерезной машины, влияющие на расход дорогостоящего алмазного инструмента и удельное энергопотребление. Среди технико-экономических показателей любой камнерезной машины определяющей является производительность (так как остальные показатели во многом производны от нее). Применительно к существующим схемам управления работой КП рассмотрим методики оценки производительности, энергопотребления и расхода алмазного инструмента.

В случае применения схемы управления с постоянной скоростью подачи средняя производительность КП рассчитывается согласно работе [5]:

$$
\Pi_{V}^{\mathrm{cp}}=K_{\mathrm{M}} \cdot \Pi_{V}^{\mathrm{max}},
$$

где $K_{\mathrm{M}}<1$ - коэффициент влияния геометрии плоскости отделения монолита на среднюю производительность резания от ее максимального значения. 
Максимальная производительность определяется выражением

$$
\Pi_{V}^{\max }=\frac{N}{b \cdot 820} \cdot\left(\frac{2 \cdot N}{\mu_{\mathrm{pac}} \cdot k_{n} \cdot b \cdot\left(\pi+\varphi_{\text {A }}\right) \cdot h_{\mathrm{mp}} \cdot V_{\mathrm{p}}}\right)^{0,5}
$$

где $h_{\text {пр }}$ - высота пропила, м; $\varphi_{д}$ - дополнительный угол охвата, рад; $\mu_{\text {рас }}$ и $k_{n}-$ коэффициенты распиловки и прерывистости режущей поверхности; $b$ - диаметр режущей втулки, м; $V_{\mathrm{p}}-$ скорость распиловки (скорость движения гибкого режущего органа), м/с.

Для продольного пропила $\left(k_{\phi}=L / H>1\right), h_{\text {пр }}=H_{y}$, а значение коэффициента $K_{\mathrm{m}}^{\text {пр }}$ рассчитывается по формуле

$$
K_{\mathrm{M}}^{\mathrm{np}}=k_{\phi} /\left(k_{\phi}+0,75-0,643 \cdot\left(D_{\text {шк }} / H_{\mathrm{y}}\right)\right)
$$

где $D_{\text {шк }}-$ диаметр ведущего шкива, м; $H_{\text {у }}$ высота уступа, м.

Для поперечного пропила, отделяющего монолит камня от массива, выполняется условие $k_{\phi}=B / H \leq 1$. В этом случае максимальная высота пропила не равна высоте уступа и находится из уравнения

$$
h_{\text {пр }}=H_{\mathrm{y}} \cdot k_{\phi} \cdot\left(2-k_{\phi}-\frac{D_{\text {шк }}}{2 \cdot H_{\mathrm{y}}}\right) /\left(1-\frac{D_{u \kappa}}{2 \cdot H_{\mathrm{y}}}\right)
$$

что в итоге определяет расчетную величину коэффициента $K_{\mathrm{M}}{ }^{\text {п } д л я ~ п о п е р е ч н о г о ~ п р о п и-~}$ ла:

$$
K_{\mathrm{M}}^{\Pi}=\left(2-\frac{D_{\mathrm{m \kappa}}}{H_{\mathrm{y}}}\right) /\left[k_{\phi}+0,75-0,643 \cdot \frac{D_{\mathrm{m \kappa}}}{H_{\mathrm{y}}}\right] .
$$

Зависимость производительности КП, соответствующей схеме управления ею в режиме постоянной мощности резания, когда $k_{\phi}=B / H \leq 1$, определяется по выражению

$$
\Pi_{N}=\frac{N}{820 \cdot b} \cdot\left(\frac{2 \cdot N}{\mu_{\mathrm{pac}} \cdot k_{n} \cdot b \cdot V_{\mathrm{p}}}\right)^{0,5} \cdot\left[\left(\frac{1}{\pi \cdot D_{\mathrm{IK}}}\right)^{0,5}-\left(\frac{1}{\left(\pi+\varphi_{\mathrm{\mu}}\right) \cdot h_{\mathrm{mp}}}\right)^{0,5}\right] .
$$

При отделении монолита по продольной плоскости $\left(k_{\phi}=L / H>1, h_{\text {пр }}=H_{y}\right)$ необходимо учитывать стационарность процесса пиления по отношению к длине контакта инструмента с породой. В этом случае производительность в режиме постоянной мощности находится как средневзвешенная величина

$$
\Pi_{N}^{\mathrm{cp}}=\left(\Pi_{N} \cdot t_{\mathrm{нест}}+\Pi_{V}^{\max } \cdot t_{\mathrm{cт}}\right) /\left(t_{\text {нест }}+t_{\mathrm{cr}}\right),
$$

где $t_{\text {нест }}=\left(k_{\phi} \cdot H_{\mathrm{y}}^{2}-0,5 \cdot\left(k_{\phi}-1\right) \cdot H_{\mathrm{y}}^{2}\right) / \Pi_{N}-$ время нестационарного пиления плоскости отделения, ч; $t_{\text {ст }}=\left(0,5 \cdot\left(k_{\phi}-1\right) \cdot H_{\mathrm{y}}^{2}\right) / \Pi_{V}^{\max }$ - время стационарного пиления плоскости отделения, ч.

Отношение производительностей при двух режимах для $k_{\phi} \leq 1$ запишется в виде

$$
\frac{\Pi_{N}}{\Pi_{V}}=\frac{1}{K_{\mathrm{M}}^{\mathrm{n}}} \cdot\left[\left(\frac{\left(\pi+\varphi_{\mathrm{z}}\right) \cdot h_{\mathrm{mp}}}{\pi \cdot D_{\mathrm{ш \kappa}}}\right)^{0,5}-1\right],
$$

а для $k_{\phi}>1$ 


$$
\frac{\Pi_{N}^{\mathrm{cp}}}{\Pi_{V}^{\mathrm{cp}}}=\frac{\left(2 \cdot k_{\phi} \cdot \Pi_{N}\right)}{K_{\mathrm{M}}^{\mathrm{mp}} \cdot\left(\left(k_{\phi}+1\right) \cdot \Pi_{V}^{\mathrm{max}}+\left(k_{\phi}-1\right) \cdot \Pi_{N}\right)} .
$$

На рис. 1 представлено отношение производительностей резания КП (9), (10), управляемой по рассмотренным выше силовым режимам.

При этом, начиная с высоты уступа 2-4 м, производительность резания в режиме $N=$ const по отношению к режиму $V_{I}=$ const возрастает по степенной зависимости в соответствии с формой плоскости отделения.

Для реализации алмазосберегающего режима распиловки необходимо, чтобы $\sigma_{n}^{\min } \rightarrow \sigma_{n}^{\text {on }}$ (т. е. отвечало условию минимального удельного расхода алмазного инструмента), где $\sigma_{n}^{\text {min }}$ величина контактного нормального напряжения, когда в процессе резания выдерживается постоянная скорость подачи (рис. 2).

Реализуемое в процессе резания энергопотребление $(A)$ для расчета удельных затрат по формуле (1) определяется для режима работы КП с постоянной скоростью подачи следующим образом:

$$
A_{V}=K / \sqrt{\sigma_{n}^{\min }}
$$

Для режима постоянной мощности резания удельное энергопотребление в случае $k_{\phi} \leq 1$ находится как средняя интегральная величина по следующей зависимости:

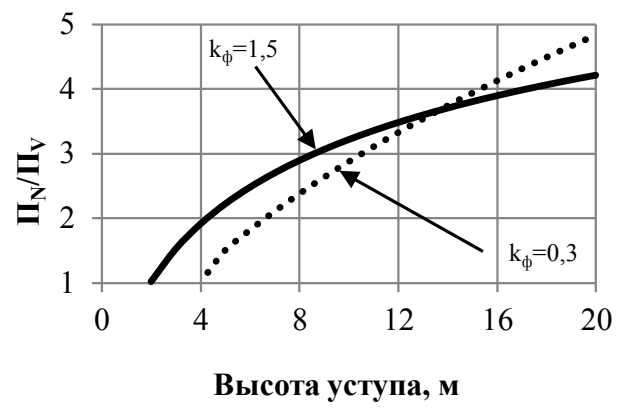

Рис. 1. Зависимость отношения производительностей резания при различных режимах работы КП от высоты уступа

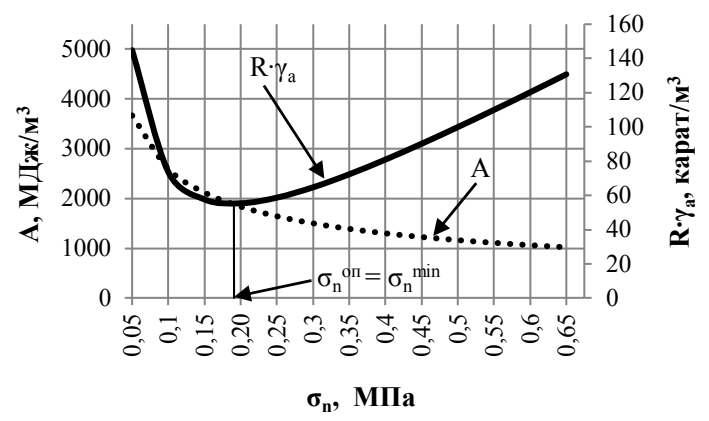

Рис. 2. Зависимости удельного расхода $(R)$ алмазного инструмента и удельной работы резания $(A)$ от нормального контактного давления $\left(\sigma_{n}\right)$ для пород с $\sigma_{c \varkappa c}=100-120$ МПа 


$$
A_{N}=\left(2 \cdot K \cdot\left(\sqrt{\sigma_{n}^{\max }}-\sqrt{\sigma_{n}^{\min }}\right)\right) /\left(\sigma_{n}^{\max }-\sigma_{n}^{\min }\right)
$$

где $\sigma_{n}^{\max }$ - максимальное значение нормального напряжения, соответствующее минимальной длине контакта инструмента с породой, Па.

При отделении монолита по продольной плоскости $\left(k_{\phi}>1\right)$ удельная работа резания в режиме постоянной мощности вычисляется как средневзвешенная величина:

$$
A_{N}^{\mathrm{cp}}=\left(A_{N} \cdot t_{\text {нест }}+A_{V} \cdot t_{\mathrm{cT}}\right) /\left(t_{\text {нест }}+t_{\mathrm{cT}}\right) \text {. }
$$

Отношение удельных работ резания при двух режимах для поперечной плоскости отделения можно записать в следующем виде:

$$
\frac{A_{N}}{A_{V}}=\frac{\left(2 \cdot \sqrt{\sigma_{n}^{\min }} \cdot\left(\sqrt{\sigma_{n}^{\max }}-\sqrt{\sigma_{n}^{\min }}\right)\right)}{\left(\sigma_{n}^{\max }-\sigma_{n}^{\min }\right)}
$$

а для продольной плоскости отделения

$$
\frac{A_{N}^{\mathrm{cp}}}{A_{V}}=\frac{\left(\sqrt{\sigma_{n}^{\min }} \cdot\left(A_{N} \cdot t_{\mathrm{Hест}}+A_{V} \cdot t_{\mathrm{cT}}\right)\right)}{\left(K \cdot\left(t_{\mathrm{Hec}}+t_{\mathrm{cT}}\right)\right)} .
$$

На рис. 3 в графическом виде показаны зависимости (14) и (15). Расчеты в данном случае выполнены при следующих данных: $N=25,47$ кВт, $\mu_{p a c}=0,25, k_{n}=0,16, b=0,01 \mathrm{M}, D_{\text {шк }}=0,8$ м, $V_{\mathrm{p}}=30 \mathrm{M} / \mathrm{c}$.

Средняя удельная работа резания (в режиме $N=$ const) по отношению к удельной работе резания, соответствующей $\sigma_{n}^{\min }=\sigma_{n}^{\text {оп }}$ (в режиме $V_{\Pi}=$ const), возрастает в соответствии с формой плоскости отделения.

Удельный расход алмазного инструмента $(R)$, как и удельная работа резания $(A)$, может быть получен на основе стендовых испытаний. Для дальнейших расчетов были приняты экспериментальные данные К.Г. Лусиняна по резанию алмазным инструментом природного камня различной прочности [6]. В результате обработки экспериментальных точек получена следующая аппроксимирующая зависимость $R$ от $\sigma_{n}$ :

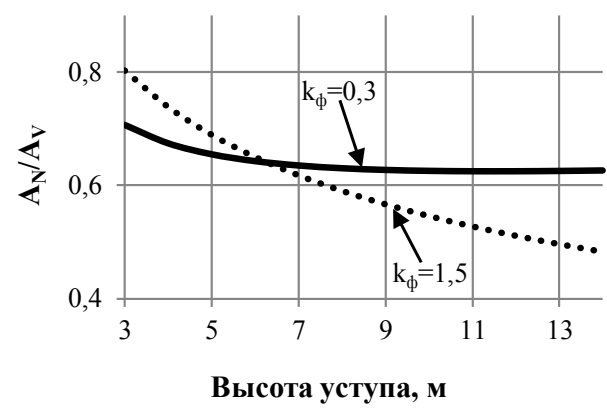

Рис. 3. Зависимость отношения среднего (в режиме $N=$ const) и соответствующего $\sigma_{n}^{\min }=\sigma_{n}^{\text {oп }}$ (в режиме $V_{\Pi}=$ const) значений удельной работы резания от высоты уступа 


$$
R=r_{0}+\frac{r_{1}}{\sigma_{n}}+r_{2} \cdot \sigma_{n}
$$

решение которой при условии $\partial R / \partial \sigma_{n}=0$ дает оптимальное контактное давление $\sigma_{n}^{\text {оп }}=\sqrt{r_{1} / r_{2}}$. Его подстановка в (16) и определяет расход алмазного инструмента в режиме $V_{\Pi=}$ const:

$$
R_{V}^{\min }=r_{0}+\frac{r_{1}}{\sqrt{r_{1} / r_{2}}}+r_{2} \cdot \sqrt{r_{1} / r_{2}}
$$

Численные значения аппроксимирующих коэффициентов $r_{0}=-1,46 \cdot 10^{-5}, r_{1}=0,376 \cdot 10^{-5} \mathrm{MПа,}$ $r_{2}=10,5 \cdot 10^{-5} 1 /$ МПа получены при резании природного камня с $\sigma_{\text {сж }}=120-140$ Мпа [7-14]. Для режима $N=$ const удельный расход может быть получен как средняя интегральная величина:

$$
R_{N}=\frac{r_{0} \cdot\left(\sigma_{n}^{\max }-\sigma_{n}^{\min }\right)+r_{1} \cdot \ln \frac{\sigma_{n}^{\max }}{\sigma_{n}^{\min }}+0,5 \cdot r_{2} \cdot\left(\left(\sigma_{n}^{\max }\right)^{2}-\left(\sigma_{n}^{\min }\right)^{2}\right)}{\sigma_{n}^{\max }-\sigma_{n}^{\min }} .
$$

Тогда их отношение для поперечной плоскости отделения запишем в следующем виде:

$$
\frac{R_{N}}{R_{V}^{\min }}=\frac{r_{0} \cdot\left(\sigma_{n}^{\max }-\sigma_{n}^{\min }\right)+r_{1} \cdot \ln \frac{\sigma_{n}^{\max }}{\sigma_{n}^{\min }}+0,5 \cdot r_{2} \cdot\left(\left(\sigma_{n}^{\max }\right)^{2}-\left(\sigma_{n}^{\min }\right)^{2}\right)}{\left(\sigma_{n}^{\max }-\sigma_{n}^{\min }\right) \cdot\left(r_{0}+\frac{r_{1}}{\sqrt{r_{1} / r_{2}}}+r_{2} \cdot \sqrt{r_{1} / r_{2}}\right)} .
$$

При отделении монолита по продольной плоскости удельный расход инструмента в режиме постоянной мощности находят как средневзвешенную величину:

$$
R_{N}^{\mathrm{cp}}=\left(R_{N} \cdot t_{\text {нест }}+R_{V}^{\min } \cdot t_{\text {ст }}\right) /\left(t_{\text {нест }}+t_{\text {ст }}\right) .
$$

Тогда отношение удельных расходов инструмента при двух режимах для продольной плоскости отделения будет таким:

$$
\frac{R_{N}^{\mathrm{cp}}}{R_{V}^{\min }}=\frac{R_{N} \cdot t_{\mathrm{HecT}}+R_{V}^{\min } \cdot t_{\mathrm{cT}}}{\left(t_{\text {нест }}+t_{\mathrm{cr}}\right) \cdot\left(r_{0}+\frac{r_{1}}{\sqrt{r_{1} / r_{2}}}+r_{2} \cdot \sqrt{r_{1} / r_{2}}\right)} .
$$

На рис. 4 в графическом виде показаны зависимости (20) и (21) при различном значении коэффициента $k_{\phi}$

Среднее значение удельного расхода алмазного инструмента (в режиме $N=$ const) по отношению к минимальному (в режиме $V_{\Pi}=$ const) возрастает в соответствии с формой плоскости отделения.

Экономические показатели при различных параметрах и режимах распиловки алмазноканатным инструментом гранодиорита Нижне-Санарского месторождения (линейные размеры монолита: $H_{\mathrm{M}}=5,6 \mathrm{M}, L_{\mathrm{M}}=8,4 \mathrm{M}, B_{\mathrm{M}}=1,7 \mathrm{м}$ ) приведены в табл. 1,2 .

Время, затраченное на резание плоскостей, для условий юго-восточного участка НижнеСанарского месторождения гранодиоритов в случае работы КП в режиме $N=$ const в 1,5 раза меньше, чем в режиме $V_{\text {П }}$ const. Результаты оценки доли пиления во всем процессе подготовки камня к выемке в зависимости от режима работы КП приведены в табл. 3. 


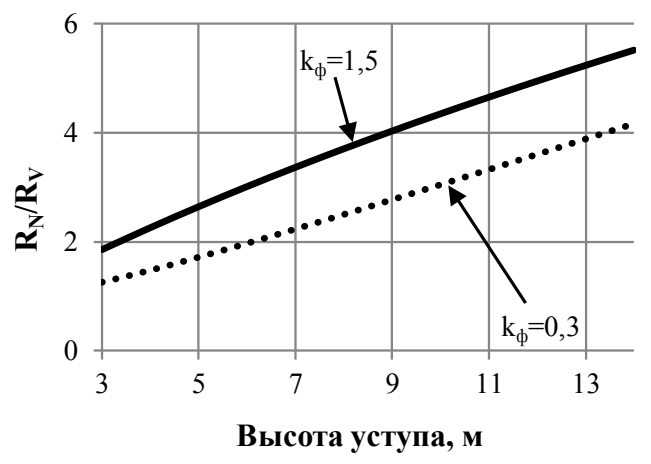

Рис. 4. Зависимость отношения среднего (в режиме $N=$ const) и минимального ( $V_{\Pi}=$ const) значений удельного расхода алмазного инструмента от высоты уступа

Таблица 1. Расчетные параметры и технико-экономические показатели работы КП в режиме VП = const

\begin{tabular}{|c|c|c|c|}
\hline Режим & \multicolumn{3}{|c|}{$V_{\Pi=}$ const,$\sigma_{n}=$ const $, \sigma_{n}{ }^{\min }=0,2 \mathrm{M \Pi а}, A=1833,58 \mathrm{MДж/ \textrm {M } ^ { 3 }}$} \\
\hline Вид плоскости отделения $\left(k_{\phi}\right)$ & Горизонтальная $(0,2)$ & Поперечная $(0,3)$ & Продольная $(1,5)$ \\
\hline$N^{\max }$, кВт & 8,24 & 12,18 & 25,47 \\
\hline$R^{\min .} \gamma_{\mathrm{a}}$, карат/м ${ }^{3}$ & \multicolumn{3}{|c|}{55,31} \\
\hline$K_{\mathrm{M}}$ & 0,63 & 0,60 & 0,70 \\
\hline$\Pi^{\max }, \mathrm{M}^{2} / \mathrm{ч}$ & 1,62 & 2,39 & 5,00 \\
\hline$\Pi^{c p}, \mathrm{M}^{2} / \mathrm{ч}$ & 1,01 & 1,42 & 3,48 \\
\hline$N_{\text {уд }}, \mathrm{\kappa BT} \cdot ч / \mathrm{M}^{2}$ & \multicolumn{3}{|c|}{18,34} \\
\hline$C_{\mathrm{AO}+3 \Pi}, \mathrm{py \sigma} / \mathrm{M}^{2}$ & 391,69 & 279,69 & 114,36 \\
\hline$C_{\text {ээ, }}$, руб/м² & \multicolumn{3}{|c|}{15,80} \\
\hline$C_{\mathrm{Aи}}, \mathrm{py \sigma} / \mathrm{M}^{2}$ & \multicolumn{3}{|c|}{158,07} \\
\hline$C_{S}, \mathrm{py \sigma} / \mathrm{M}^{2}$ & 565,56 & 453,56 & 288,23 \\
\hline$S, \mathrm{M}^{2}$ & 14,11 & 9,41 & 47,04 \\
\hline$t_{\text {пиления, }}$, ч & 14,1 & 6,7 & 13,5 \\
\hline$\sum t_{\text {пиления, }}, \mathbf{ч}$ & \multicolumn{3}{|c|}{34,4} \\
\hline$C$, тыс. руб. & 7,98 & 4,27 & 13,56 \\
\hline$\sum C$ (по монолиту), тыс. руб. & \multicolumn{3}{|c|}{25,81} \\
\hline
\end{tabular}

Таким образом, время, затраченное на выполнение пропилов КП, в процессе подготовки к выемке камня занимает 51,5 и $61,5 \%$ для режимов $N=$ const и $V_{\Pi=}$ const соответственHO.

По формуле (1) были рассчитаны удельные эксплуатационные затраты на резание плоскостей отделения монолита от массива. Это позволило представить в графическом виде отношения удельных эксплуатационных затрат при различных режимах работы КП от высоты уступа (рис. 5).

Удельные эксплуатационные затраты на резание плоскостей в режиме $N=$ const по отношению к режиму $V_{\Pi=}$ const возрастают начиная с высоты уступа 4,5 м (рис. 5,6$)$ в соответствии 
Таблица 2. Расчетные параметры и технико-экономические показатели работы КП в режиме $N=$ const

\begin{tabular}{|c|c|c|c|}
\hline Режим & \multicolumn{3}{|c|}{$N=$ const,$\sigma_{n}=$ var } \\
\hline Вид плоскости отделения $\left(\mathrm{k}_{\phi}\right)$ & Горизонтальная $(0,2)$ & Поперечная $(0,3)$ & Продольная $(1,5)$ \\
\hline$N$, кВт & 8,24 & 12,18 & 25,47 \\
\hline$\sigma_{\mathrm{n}}^{\max }, \mathrm{M \Pi а}$ & 0,57 & 0,81 & 1,69 \\
\hline$A^{c p}, \mathrm{MДж/ \textrm {M } ^ { 3 }}$ & 1364,34 & 1219,42 & 1185,90 \\
\hline$R \cdot \gamma_{\mathrm{a}}$, карат $/ \mathrm{M}^{3}$ & 78,21 & 103,20 & 158,61 \\
\hline$\Pi, \mathrm{M}^{2} / \mathrm{ч}$ & 1,10 & 2,41 & 8,28 \\
\hline$N_{y \partial}$, кВт・ч/ $/ \mathrm{M}^{2}$ & 13,64 & 12,19 & 11,86 \\
\hline$C_{A O+3 \Pi}, \mathrm{py} \sigma / \mathrm{m}^{2}$ & 362,96 & 165,00 & 47,99 \\
\hline 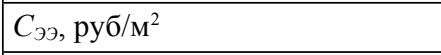 & 11,75 & 10,51 & 10,22 \\
\hline$C_{A И}, \mathrm{py} \sigma / \mathrm{M}^{2}$ & 223,54 & 294,94 & 453,30 \\
\hline$C_{S}$, руб/ $/ \mathrm{m}^{2}$ & 598,26 & 470,45 & 511,51 \\
\hline$t_{\text {пиления }}, \mathrm{ч}$ & 13,0 & 4,0 & 5,7 \\
\hline$\sum t_{\text {пиления }}$, ч & \multicolumn{3}{|c|}{22,7} \\
\hline$C$, тыс. руб. & 8,44 & 4,43 & 24,06 \\
\hline$\sum C$ (по монолиту), тыс. руб. & \multicolumn{3}{|c|}{36,93} \\
\hline
\end{tabular}

Таблица 3. Доля времени выполнения пропилов КП в процессе подготовки к выемке гранодиорита Нижне-Санарского месторождения при различных режимах резания

\begin{tabular}{|c|c|c|}
\hline \multirow{2}{*}{ Вид работ } & \multicolumn{2}{|c|}{ Время выполнения, ч } \\
\hline & $V_{\Pi=}$ const, $\sigma_{n=}$ const & $N=$ const,$\sigma_{n}=\operatorname{var}$ \\
\hline Бурение горизонтальной продольной скважины $(8,4$ м) & \multicolumn{2}{|c|}{3,5} \\
\hline Поиск отметок для стыковки скважин & \multicolumn{2}{|c|}{3,0} \\
\hline Бурение горизонтальной поперечной скважины (1,7 м) & \multicolumn{2}{|c|}{2,0} \\
\hline Горизонтальный пропил КП (14,28 м²) & 14,14 & 13,0 \\
\hline Поиск отметок для стыковки скважин & \multicolumn{2}{|c|}{3,0} \\
\hline Бурение вертикальной скважины (5,6 м) & \multicolumn{2}{|c|}{2,5} \\
\hline Вертикальный поперечный пропил КП (9,52 м²) & 6,70 & 4,0 \\
\hline Вертикальный продольный пропил КП (47,04 м²) & 13,52 & 5,68 \\
\hline Отсыпка подушки & \multicolumn{2}{|c|}{0,5} \\
\hline Опрокидывание монолита на рабочую площадку & \multicolumn{2}{|c|}{3,0} \\
\hline $\begin{array}{l}\text { Разделка опрокинутого монолита и пассировка блоков } \\
\text { шпуровым способом с применением механических клиньев }\end{array}$ & \multicolumn{2}{|c|}{4,0} \\
\hline Время подготовки к выемке блоков природного камня, ч & 55,9 & 44,1 \\
\hline Время пиления, ч (в \% от общего) & $34,4(61,5 \%)$ & $22,7(51,5 \%)$ \\
\hline
\end{tabular}

с формой плоскости отделения. Так, при высоте уступа $H_{\mathrm{y}}^{\text {оп }}=5,6$ м отношение значений себестоимости $C_{s(N)} / C_{s(V)}$ составляет $1,0-1,8$ в зависимости от величины коэффициента $k_{\phi}$.

Основным результатом проведенных исследований и полученных зависимостей (1), (9), (10), (14), (15), (19), (21) влияния силового режима на технико-экономические показатели явля- 


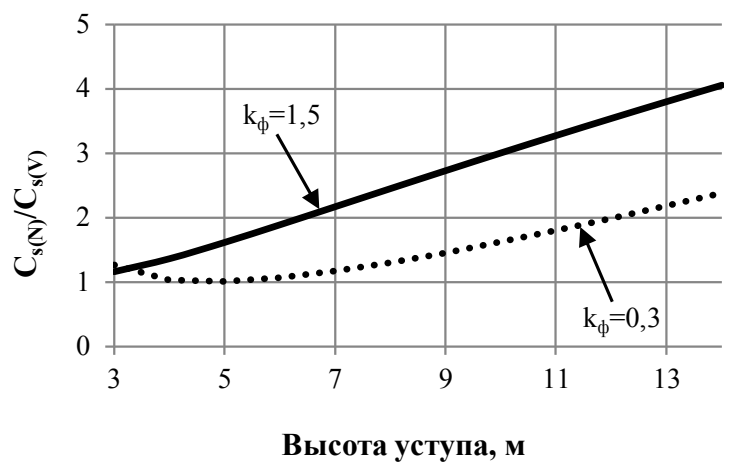

Рис. 5. Зависимость отношения удельных эксплуатационных затрат на резание плоскостей при различных режимах работы КП от высоты уступа

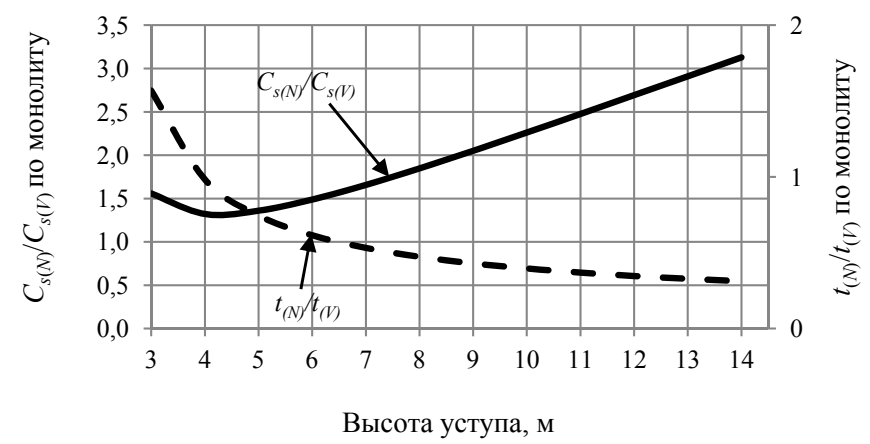

Рис. 6. Зависимость отношения удельных эксплуатационных затрат на резание плоскостей и времени, затраченного на пиление (по монолиту) при различных режимах работы КП от высоты уступа

ется вывод о неоднозначном вкладе в эксплуатационные затраты производительности, энергопотребления и расхода алмазного инструмента при резании. Так как силовой режим в пределах заданной мощности главного привода канатной пилы связан с высотой пропила (уступа), то с практических позиций целесообразнее полученные технико-экономические показатели представлять в виде функциональных зависимостей от высоты уступа для различных режимов работы КП.

Анализ таких итоговых показателей, как производительность отделения монолита от массива (рис. 7a) и эксплуатационные затраты на отделение (рис. 7б) в зависимости от высоты уступа для различных режимов работы КП, не позволяет выделить преимущества одного режима работы КП от другого, так как повышение производительности отделения монолита сопровождается повышением эксплуатационных затрат на его отделение при работе в режиме постоянной мощности резания и, наоборот, когда снижению эксплуатационных затрат соответствует понижение производительности, если КП работает в режиме постоянной скорости подачи.

В этом случае корректная оценка зависимости режима работы КП от высоты уступа может быть дана на основе комплексного технико-экономического показателя:

$$
-937-
$$




$$
c_{w}=\sum C /(W / t)
$$

где $\Sigma C$ - эксплуатационные затраты на отделение монолита от массива, тыс. руб.; $W / t$ - производительность отделения монолита от массива, $\mathbf{M}^{3} /$ ч

Тогда выбор режима работы КП в зависимости от высоты уступа осуществляется по минимальной величине предложенного комплексного показателя (рис. 7в).

Как видно из рис. 7в, при отделении монолита с высотой добычного уступа менее 4,5 м предпочтение следует отдавать режиму с постоянной скоростью подачи КП на забой. При высоте уступа более 4,5 м, когда показатель $c_{w}$ изменяется незначительно от режима работы КП, целесообразен режим работы с постоянной мощностью резания, обеспечивающий более высокую производительность отделения монолита.

a

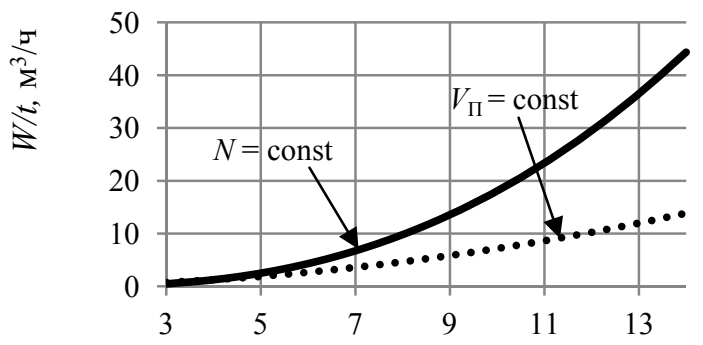

Высота уступа, м

б

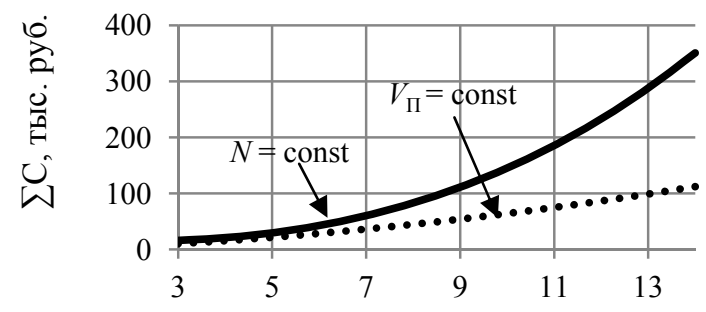

Высота уступа, м

B

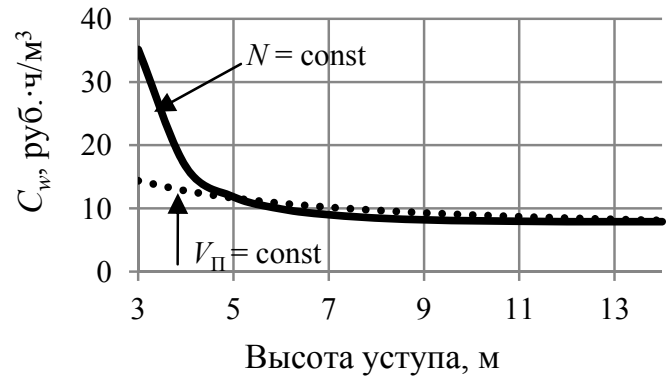

Рис. 7. Зависимость от высоты уступа (при различных режимах работы КП): а - производительности отделения монолита от массива с помощью КП (W/t, $\left.\mathrm{M}^{3} / \mathrm{ч}\right) ;$ б - эксплуатационных затрат на отделение монолита от массива ( $\sum \mathrm{C}$, тыс. руб.); в - комплексного технико-экономического показателя $\left(C_{w}\right.$, руб.ч $\left./ \mathrm{M}^{3}\right)$ 


\section{Выводы}

1. Получены зависимости отношения производительностей резания при различных режимах работы КП от высоты уступа. При этом, начиная с высоты уступа 2-4 м, производитель-

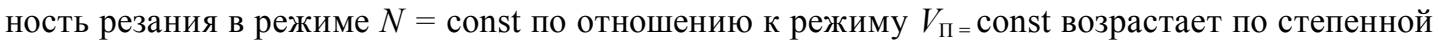
зависимости в соответствии с формой плоскости отделения.

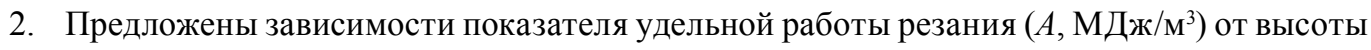
уступа при различных схемах управления КП и величине коэффициента $k_{\phi}$. Средняя удельная работа резания (в режиме $N=$ const) по отношению к удельной работе резания, соответствующей $\sigma_{n}^{\min }=\sigma_{n}^{\text {oп }}$ (в режиме $V_{\Pi=}$ const), возрастает в соответствии с формой плоскости отделения.

3. Установлены зависимости показателя $R \cdot \gamma_{\mathrm{a}}($ карат/Mㄱ) от высоты уступа при различных схемах управления КП и величине коэффициента $k_{\phi}$. Среднее значение удельного расхода алмазного инструмента (в режиме $N=$ const) по отношению к минимальному (в режиме $V_{\Pi}=$ const) возрастает в соответствии с формой плоскости отделения.

4. Время, затраченное на резание плоскостей, для условий юго-восточного участка Нижне-Санарского месторождения гранодиоритов в случае работы КП в режиме $N=$ const $\left(22,7\right.$ ч) в 1,5 раза меньше, чем в режиме $V_{\text {П= }}$ const $(34,4$ ч). При этом доля времени, затраченного на выполнение пропилов КП, в процессе подготовки к выемке камня занимает 51,5 и 61,5 \% для режимов $N=$ const и $V_{\Pi=}$ const соответственно.

5. Удельные эксплуатационные затраты на резание плоскостей в режиме $N=$ const по отношению к режиму $V_{\Pi=}$ const возрастают в соответствии с формой плоскости отделения. Отношение значений удельных эксплуатационных затрат $C_{s(N)} / C_{s(V)}$ при высоте уступа $H_{\mathrm{y}}^{\text {oп }}=5,6 \mathrm{м}$ составляет 1,0-1,8 в зависимости от величины коэффициента $k_{\phi}$.

6. Производительность отделения монолита от массива с помощью КП, работающей в режиме постоянной мощности резания, при высоте уступа 5-13 м повышается в 1,2-3 раза по отношению к режиму постоянной скорости подачи КП на забой. Одновременно повышаются во столько же раз и эксплуатационные затраты на отделение монолита, что не дает основания по данным показателям выявить преимущества одного режима от другого. Поэтому за критерий оценки принимается комплексный технико-экономический показатель $\left(c_{w}\right)$, характеризуемый затратами, отнесенными к интенсивности отделения объемов камня от массива. Выбор рационального режима работы КП от высоты уступа достигается путем минимизации величины этого комплексного показателя.

7. Для отделения монолитов камня от породного массива при высоте добычного уступа менее 4,5 м следует выбирать режим резания с постоянной скоростью подачи КП на забой. При высоте уступа более 4,5 м, когда показатель $C_{w}$ изменяется незначительно в зависимости от режима работы КП, целесообразен режим работы с постоянной мощностью резания, обеспечивающий более высокую производительность отделения монолита.

\section{Список литературы}

[1] Першин Г.Д., Уляков М.С. // Физико-технические проблемы разработки полезных ископаемых. 2014. № 2. С. 125-135.

[2] Першин Г.Д., Уляков М.С. // Вестник МГТУ. 2014. № 4 (48). С. 14-21. 
[3] Першин Г.Д., Уляков М.С. // Известия высших учебных заведений. Горный журнал. 2014. № 7. C. 10-18.

[4] Першин Г.Д., Уляков М.С. // Известия вузов. Горный журнал. 2014. № 8. С. 22-30.

[5] Першин Г.Д., Пшеничная Е.Г., Уляков М.С. // Добыча, обработка и применение природного камня: сб. науч. тр. Магнитогорск: Изд-во МГТУ, 2012. С. 54-63.

[6] Акопян Р.В., Лусинян К.Г. // Изучение природных каменных материалов и силикатного сырья, разработка эффективной техники и технологии производства: сб. науч. тр. Ереван: НИИКС, 1983. С. 40-49.

[7] Пащенко К.Г. Бахматов Ю.Ф., Кальченко А.А. и др. // Успехи современного естествознания. 2014. № 12-4. С. 421-424.

[8] Бахматов Ю.Ф. Пащенко К.Г., Кальченко А.А. и др. // Международный журнал прикладных и фундаментальных исследований. 2014. № 12-1. С. 8-11.

[9] Извеков Ю.А. // Молодой ученый. 2014. № 4. С. 179-182.

[10] Чалкова К.Д. // Молодой ученый. 2014. № 21 (80). С. 243-244.

[11] Вагин В.С., Филатов А.М., Курочкин А.И. // Вестник МГТУ. 2014. № 3 (47). С. 25-29.

[12] Бахматов Ю.Ф. Пащенко К.Г., Кальченко А.А. и др. // Металлург. 2014. № 4. С. 88-91.

[13] Левиџький В.Г., Соболевський Р.В. // Восточно-Европейский журнал передовых технологий. 2014. Т. 3. № 3 (69). С. 48-52.

[14] Айбашев Д.М., Кольга А.Д., Хажиев А.А. // Вестник развития науки и образования. 2014. № 3. С. 163-166. 